\title{
Laparoscopic Ovarian Cystectomy in a Very Low-Birth Weight Preterm Newborn
}

\author{
Valentina Pastore, MD, Angela Basile, MD, Raffaella Cocomazzi, MD, Fabio Bartoli, MD \\ Pediatric Surgery Unit, University of Foggia, Foggia, Italy (all authors).
}

\begin{abstract}
Introduction: Minimally invasive surgery is normally used in children but is not so common in newborns, especially those with very low birth weight, as a consequence of an immature cardiopulmonary system and technical difficulties.

Case Description: A preterm female with very low birth weight $(1.4 \mathrm{~kg})$, born in the $32 \mathrm{nd}$ week of gestation with a prenatal diagnosis of abdominal cyst, presented with distension of the abdomen and a palpable tension mass in the right iliac and hypochondriac region on the first day of postnatal life. Clinical investigation findings indicated a suspected simple right-side ovarian cyst measuring $5.4 \times 8.55 \times 8.72 \mathrm{~cm}$ occupying most of the abdomen. The patient's clinical condition deteriorated quickly with respiratory distress due to the mass's effect. The cyst was surgically aspirated, isolated, and removed by a completely laparoscopic approach. The operating time was 30 minutes, the respiratory distress resolved immediately, and the patient's recovery was uneventful.

Discussion: Ovarian cysts are the most common ovarian lesions in newborns; they are usually simple and benign but can be complicated and symptomatic. Minimally invasive surgery is nowadays the gold standard for newborns and children with ovarian cysts, even when complications occur. Furthermore, we have shown that laparoscopy also can be used safely in distressed preterm very low-birth weight newborns without cardiorespiratory consequences, anesthetic incidents, or hypothermia.
\end{abstract}

Key Words: Ovarian cyst, VLBW, Laparoscopy.

Citation Pastore V, Basile A, Cocomazzi R, Bartoli F. Laparoscopic ovarian cystectomy in a very low-birth weight preterm newborn. CRSLS e2014.00219. DOI 10.4293/CRSLS.2014.00219

Copyright $\odot 2014$ SLS This is an open-access article distributed under the terms of the Creative Commons Attribution-Noncommercial-ShareAlike 3.0 Unported license, which permits unrestricted noncommercial use, distribution, and reproduction in any medium, provided the original author and source are credited.

Address correspondence to: Valentina Pastore, Pediatric Surgery Unit, University of Foggia, Viale Pinto 1, 71122, Foggia, Italy. Telephone: 00390881732541, Fax: 00390881732541, E-mail: valentina.pastore@teletu.it

\section{INTRODUCTION}

In the past decade, after the success of minimally invasive surgical techniques in children, the application of these techniques in newborns was a logical next step. However, the use of these techniques in newborns has spread slowly because of the need to downsize surgical instruments, the relatively long learning curve, and the need for reliable anesthetic procedures ensuring good tolerance to pneumoperitoneum. Nevertheless, the potential impact of carbon dioxide pneumoperitoneum on the immature neonatal cardiopulmonary system is a matter of great concern. In fact, the peritoneal absorption surface per unit of weight is higher in newborns. The low quantity of peritoneal fat and minimal distance between vessels and the serous surface increase the permeability of the perito- neum to carbon dioxide. ${ }^{1}$ In addition, recent improvements in imaging techniques and a trend toward routine antenatal ultrasonographic scanning have increased the frequency of intrauterine diagnosis of many congenital defects and malformations (especially abdominal cysts), requiring more extensive use of laparoscopy even in newborns. The differential diagnosis for a cystic mass in the female fetus should consider intestinal duplication; hydronephrosis; meconium; mesenteric, urachal, omental, choledochal, or ovarian cyst; or only bladder distension. ${ }^{2}$

We report the case of a preterm female with very low birth weight (VLBW) born in the 32nd week of gestation with a cystic lesion in the abdomen who underwent laparoscopy for abdominal distension and respiratory distress. 


\section{CASE DESCRIPTION}

A female newborn weighing $1.4 \mathrm{~kg}$, with a prenatal diagnosis (30th week of gestation) of abdominal cyst measuring $8.5 \times 8 \mathrm{~cm}$, was delivered preterm by caesarian section in the $32 \mathrm{nd}$ week of gestation. On the first day of life, the newborn presented with distension of the abdomen and a tense mass was palpated in the right iliac and hypochondriac region. Ultrasonography showed an anechoic thin-walled cyst measuring $5.4 \times 8.55 \times 8.72 \mathrm{~cm}$ on the right side of the pelvis, with no internal echoes or calcification. A computed tomography scan without contrast showed a large well-defined hypodense lesion, without septations, measuring $5.5 \times 8.60 \times 9 \mathrm{~cm}$ and suggesting a diagnosis of a simple right ovarian cyst. The findings of all the routine hematologic and biochemical investigations were normal, and there were no other associated anomalies.

As the hours passed, severe respiratory distress developed, requiring assisted ventilation, probably due to the mass's effect on the diaphragm; thus laparoscopy was started. The cyst was surgically aspirated, and laparoscopy was started with placement of one 5-mm Hasson port for the camera in the xiphoid region and two 3-mm trocars for manipulation in the epigastric regions. The newborn did not tolerate pneumoperitoneum $>3 \mathrm{~mm} \mathrm{Hg}$ because of the severe drop in oxygen saturation. The cyst was grasped, stitched to the abdominal wall, and then easily dissected from the remaining ovarian tissue. The cyst was extracted through the Hasson port. A Penrose drain was left in situ for 1 day. The left ovary had a small cyst that was left untouched. The operating time was 30 minutes. The newborn had an uneventful recovery with a rapid decrease in respiratory distress. She started feeding 24 hours later. Histologic examination showed a follicular ovarian cyst; the ovarian fluid showed no malignant cells. Follow-up ultrasonography after 6 months showed normal findings.

\section{DISCUSSION}

Cysts are the most common ovarian masses found in female fetuses and newborns. The first neonatal ovarian cyst was reported in 1889 as an autopsy finding in a stillborn preterm infant. ${ }^{3}$ In 1942 Bulfamonte reported the first case of an ovarian cyst successfully treated in a newborn, ${ }^{4}$ whereas the first prenatal detection of a fetal ovarian cyst was reported in 1975 by Valenti et al. ${ }^{5}$ It is generally accepted that in the newborn, the term "pathological cyst" refers to a cyst with a diameter $>2 \mathrm{~cm}$. The classification of neonatal cysts of Nussbaum et $\mathrm{al}^{6}$ is divided into simple or uncomplicated and complex or com- plicated cysts suggesting torsion. The primary structures of simple cysts are functional and are also called "follicular cysts"; they are almost always unilocular, seen in microscopic sections as follicle cysts surrounded by granulosa epithelial cells. The term "complex cyst" is used for a thick-walled septated cyst that contains a blood clot or debris. ${ }^{6}$

Cysts occur because of hormonal stimulation during either the intrauterine or perinatal period. The incidence of neonatal ovarian cysts rises with increasing placental chorionic gonadotropin levels in complicated pregnancies with large placenta, such as in cases of diabetes, pre-eclampsia, and Rh incompatibility. They also occur because of the abnormal development of the primitive gonad, probably because of disruption of its vascularization or, in preterm babies, immaturity of the hypothalamic-pituitary-gonadal axis, which causes ovarian hyperstimulation. ${ }^{7}$ In addition, fetal hypothyroidism and congenital adrenal hyperplasia due to 21 -hydroxylase deficiency or $11 \beta$-hydroxylase deficiency have been reported to cause ovarian cysts. ${ }^{8}$ The decrease in maternal-placental estrogen and $\beta$ human chorionic gonadotropin levels after birth and the infant's neurologic maturation lead to spontaneous regression of the cysts. However, because follicle-stimulating hormone and luteinizing hormone levels of infants continue to increase until the maturation of the gonadostat mechanism, cysts may continue to enlarge for about 3 months after birth. ${ }^{9}$ Spontaneous regression, which occurs in approximately $25 \%$ to $50 \%$ of cases, is more frequent with smaller cysts. Neonatal ovarian cysts may cause pain, irritability, vomiting, fever, and abdominal distension. Peritonitis, anemia due to intracystic hemorrhage, fetal tachycardia due to peritoneal irritation or anemia, and sudden infant death syndrome may also occur. Large cysts may cause intestinal and urinary obstruction because of their size and dystocia. The abdominal and thoracic mass effect gives rise to pulmonary hypoplasia and polyhydramnios. Torsion is the most common (50\%-78\%) complication especially if the cyst has a long pedicle; the ovarian size rapidly increases, and its ultrasonographic features change to complex. It frequently leads to further complications such as a rupture, resulting in hemoperitoneum, ascites, adhesion with adjacent organs causing urinary and intestinal obstruction, calcification of cyst walls, and autoamputation of the ovary. ${ }^{10}$

Preserving the ovary is the key goal in the management of these apparently benign lesions. The most appropriate clinical approach is to adopt a wait-and-see policy, assessing the course of the condition by means of periodic ultrasonographic monitoring. Symptomatic and complex cysts should 
be removed by surgery as soon as possible to avoid complications. The management options for neonatal ovarian cysts vary from antenatal transuterine aspiration to postnatal intervention in the form of repeated ultrasonography-guided needle aspiration, laparotomy, or laparoscopic oophorectomy or ovarian cystectomy. ${ }^{11}$ Recent advances in laparoscopy and neonatal anesthesia have opened up new perspectives for minimally invasive management that facilitates the demarcation of the lesion, as well as evaluation of the contralateral ovary, and allows aspiration of the cyst, cystectomy, decapsulation of the ovary, stripping of cysts, and where necessary, oophorectomy. ${ }^{12}$

In our VLBW newborn, the sudden appearance of respiratory distress and abdominal distension forced us to start surgery. We found laparoscopy very useful not just because of its combined diagnostic and therapeutic effectiveness but also because of the minimal scars and adhesion formation it entails. The only serious technical problem was the need for minimal intra-abdominal pressure, which also reduced the already poor working space. We did not observe any cardiorespiratory consequences, anesthetic incidents, or hypothermia. Furthermore, to our knowledge, this preterm VLBW neonate seems to be the smallest patient in whom a complete intraperitoneal ovarian cystectomy has been carried out laparoscopically.

\section{References:}

1. Fuentes S, Cano I, Lopez M, et al. Cardiovascular and respiratory changes during laparoscopy in neonates and infants [in Spanish]. Cir Pediatr. 2012;25:126-128.

2. Turgal M, Ozyuncu O, Yazicioglu A. Outcome of sonographically suspected fetal ovarian cysts. J Matern Fetal Neonatal Med. 2013;26(17):1728-1732.
3. Mudholkar VG, Acharya AS, Kulkarni AM, et al. Antenatally diagnosed neonatal ovarian cyst with torsion. Indian J Pathol Microbiol. 2011;54:228-229.

4. Carlson DH, Griscon T. Ovarian cysts in the newborns. Am J Roentgenol Radium Ther Nucl Med. 1972;116:664-672. With "Bulfamonte J.C. Large alarian cyst in newborn child. Am J Surg. 1942;55:175-176.

5. Valenti C, Kassner EG, Yermakov V. Antenatal diagnosis of a fetal ovarian cyst. Am J Obstet Gynecol. 1975;15:216-219.

6. Nussbaum AR, Sanders RC, Hartman DS, et al. Neonatal ovarian cyst: sonographic-pathologic correlation. Radiology. 1988;168:817-821.

7. Zampieri N, Borruto F, Zamboni C, et al. Foetal and neonatal ovarian cysts: a 5-year experience. Arch Gynecol Obstet. 2008; 277:303-306.

8. Guran T, Yesil G, Guran O, et al. A giant ovarian cyst in a neonate with classical 21-hydroxylase deficiency with very high testosterone levels demonstrating a high-dose hook effect. J Clin Res Pediatr Endocrinol. 2012;4:151-153.

9. Akin MA, Akin L, Ozbek S, et al. Fetal-neonatal ovarian cysts-their monitoring and management: retrospective evaluation of 20 cases and review of the literature. J Clin Res Pediatr Endocrinol. 2010;2:28-33.

10. Hasiakos D, Papakonstantinou K, Bacanu AM, et al. Clinical experience of five fetal ovarian cysts: diagnosis and follow-up. Arch Gynecol Obstet. 2008;277:575-578.

11. Marinkovic S, Jokic R, Bukarica S, et al. Surgical treatment of neonatal ovarian cysts. Med Pregl. 2011;64:408-412.

12. Dolgin SE. Ovarian masses in the newborn. Semin Pediatr Surg. 2000;9:121-127. 